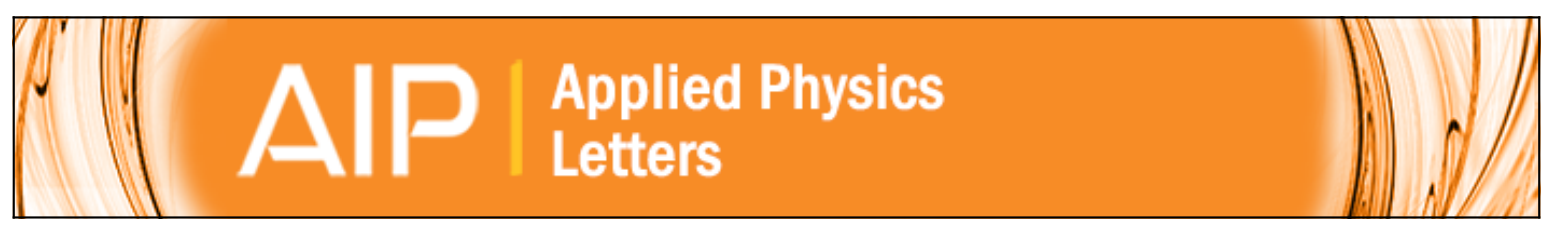

\title{
Carrier capture delay and modulation bandwidth in an edge-emitting quantum dot laser
}

Levon V. Asryan, Yuchang Wu, and Robert A. Suris

Citation: Applied Physics Letters 98, 131108 (2011); doi: 10.1063/1.3571295

View online: http://dx.doi.org/10.1063/1.3571295

View Table of Contents: http://scitation.aip.org/content/aip/journal/apl/98/13?ver=pdfcov

Published by the AIP Publishing

\section{Articles you may be interested in}

Effect of excited states on the ground-state modulation bandwidth in quantum dot lasers

Appl. Phys. Lett. 102, 191102 (2013); 10.1063/1.4804994

Influencing modulation properties of quantum-dot semiconductor lasers by carrier lifetime engineering Appl. Phys. Lett. 101, 131107 (2012); 10.1063/1.4754588

Effect of internal optical loss on the modulation bandwidth of a quantum dot laser

Appl. Phys. Lett. 100, 131106 (2012); 10.1063/1.3697683

Upper limit for the modulation bandwidth of a quantum dot laser

Appl. Phys. Lett. 96, 221112 (2010); 10.1063/1.3446968

Single-mode submonolayer quantum-dot vertical-cavity surface-emitting lasers with high modulation bandwidth

Appl. Phys. Lett. 89, 141106 (2006); 10.1063/1.2358114

\section{AlP Re-register for Table of Content Alerts}




\title{
Carrier capture delay and modulation bandwidth in an edge-emitting quantum dot laser
}

\author{
Levon V. Asryan, ${ }^{1, a)}$ Yuchang $\mathrm{Wu},{ }^{1, \mathrm{~b})}$ and Robert A. Suris ${ }^{2, \mathrm{c})}$ \\ ${ }^{1}$ Virginia Polytechnic Institute and State University, Blacksburg, Virginia 24061, USA \\ ${ }^{2}$ Ioffe Physico-Technical Institute, Saint Petersburg 194021, Russia
}

(Received 1 March 2011; accepted 8 March 2011; published online 30 March 2011)

\begin{abstract}
We show that the carrier capture from the optical confinement layer into quantum dots (QDs) can strongly limit the modulation bandwidth $\omega_{-3}$ dB of a QD laser. As a function of the cross-section $\sigma_{\mathrm{n}}$ of carrier capture into a QD, $\omega_{-3}$ dB asymptotically approaches its highest value when $\sigma_{\mathrm{n}} \rightarrow \infty$ (the case of instantaneous capture). With reducing $\sigma_{\mathrm{n}}, \omega_{-3} \mathrm{~dB}$ decreases and becomes zero at a certain nonvanishing $\sigma_{\mathrm{n}}^{\mathrm{min}}$. The use of multiple-layers with QDs significantly improves the laser modulation response- $\omega_{-3} \mathrm{~dB}$ is considerably higher in a multilayer structure as compared to a single-layer structure at the same dc current. (C) 2011 American Institute of Physics. [doi:10.1063/1.3571295]
\end{abstract}

Due to the quantum-size effect, reducing dimensionality of the active region has been a key to developing lowthreshold semiconductor lasers. ${ }^{1,2}$ In commercial diode lasers, a two-dimensional (2D) active region [quantum well $(\mathrm{QW})]$ is used. ${ }^{3,4}$ In quantum dot (QD) lasers, an ultimate case of a zero-dimensional active region is realized. ${ }^{5,6}$ The interesting physics involved and the potential for wide range of applications have motivated extensive studies of QD lasers. However, in contrast to the steady-state characteristics, the dynamic properties of QD lasers need to be further scrutinized. In particular, the potential of QD lasers for highspeed direct modulation of the output optical power by injection current should be clarified.

In Ref. 7, the highest modulation bandwidth attainable in QD lasers was estimated. For this purpose, an idealized situation of instantaneous carrier capture into QDs was assumed. In actual semiconductor lasers, carriers are not directly injected into the quantum-confined active regionthey are first injected into the optical confinement layer (OCL) and then captured into the active region (Fig. 1). Indirect injection adversely affects the laser operating characteristics - the threshold current is increased ${ }^{8}$ and more temperature-sensitive, ${ }^{9}$ and the output optical power is decreased. ${ }^{10,11}$ Due to a transport delay across the OCL and a capture delay from the OCL into the active region, the bandwidth of direct modulation of the output power by injection current is also reduced (see, e.g., Ref. 12 for QW lasers).

In this letter, we briefly report on the effect of noninstantaneous capture of carriers into QDs on the modulation bandwidth of an edge-emitting QD laser. Our model is based on the following set of three coupled rate equations for free carriers in the OCL, carriers confined in QDs, and photons:

$$
\begin{aligned}
\frac{\partial n_{\mathrm{OCL}}}{\partial t}= & \frac{j}{e b}-\sigma_{\mathrm{n}} \mathrm{v}_{\mathrm{n}} \frac{N_{\mathrm{S}}}{b}\left(1-f_{\mathrm{n}}\right) n_{\mathrm{OCL}}+\sigma_{\mathrm{n}} \mathrm{v}_{\mathrm{n}} n_{1} \frac{N_{\mathrm{S}}}{b} f_{\mathrm{n}} \\
& -B n_{\mathrm{OCL}}^{2},
\end{aligned}
$$

\footnotetext{
${ }^{a)}$ Electronic mail: asryan@vt.edu.

${ }^{\mathrm{b})}$ Electronic mail: yuchangw@vt.edu.

${ }^{c)}$ Electronic mail: suris@theory.ioffe.ru.
}

$$
\begin{aligned}
& \frac{\partial}{\partial t}\left(2 \frac{N_{\mathrm{S}}}{b} f_{\mathrm{n}}\right)= \sigma_{\mathrm{n}} \mathrm{v}_{\mathrm{n}} \frac{N_{\mathrm{S}}}{b}\left(1-f_{\mathrm{n}}\right) n_{\mathrm{OCL}}-\sigma_{\mathrm{n}} \mathrm{v}_{\mathrm{n}} n_{1} \frac{N_{\mathrm{S}}}{b} f_{\mathrm{n}} \\
&-\frac{N_{\mathrm{S}}}{b} \frac{f_{\mathrm{n}}^{2}}{\tau_{\mathrm{QD}}}-\mathrm{v}_{\mathrm{g}} g^{\max }\left(2 f_{\mathrm{n}}-1\right) n_{\mathrm{ph}}, \\
& \frac{\partial n_{\mathrm{ph}}}{\partial t}=\mathrm{v}_{\mathrm{g}} g^{\max }\left(2 f_{\mathrm{n}}-1\right) n_{\mathrm{ph}}-\mathrm{v}_{\mathrm{g}} \beta n_{\mathrm{ph}},
\end{aligned}
$$

where $n_{\mathrm{OCL}}$ is the free carrier density in the OCL, $j$ is the injection current density, $b$ is the OCL thickness, $\sigma_{\mathrm{n}}$ is the cross-section of carrier capture into a $\mathrm{QD}, \mathrm{v}_{\mathrm{n}}$ is the carrier thermal velocity, $N_{\mathrm{S}}$ is the surface density of QDs, $f_{\mathrm{n}}$ is the occupancy of the energy-level of a carrier confined in a QD, $B$ is the spontaneous radiative recombination constant for the OCL, $\tau_{\mathrm{QD}}$ is the spontaneous radiative time in a $\mathrm{QD}, \mathrm{v}_{\mathrm{g}}$ is the group velocity of light, $g^{\max }$ is the maximum modal gain, ${ }^{8}$ $n_{\mathrm{ph}}$ is the photon density (per unit volume of the OCL) in the lasing mode, $\beta=(1 / L) \ln (1 / R)$ is the mirror loss, $L$ is the cavity length, and $R$ is the facet reflectivity.

In Eqs. (1) and (2), the quantity $n_{1}=N_{\mathrm{c}}^{3 \mathrm{D}} \exp \left(-E_{\mathrm{n}} / T\right)$ characterizes the carrier thermal escape from a QD to the OCL, where $N_{\mathrm{c}}^{3 \mathrm{D}}$ is the effective density of states in the OCL, $E_{\mathrm{n}}$ is the carrier thermal excitation energy from a $\mathrm{QD}$, and $T$ is the temperature (in units of energy).

Strictly speaking, $\sigma_{\mathrm{n}}$ is the only parameter adequately describing the capture/escape into/from a QD. Using $\sigma_{\mathrm{n}}$, two distinct characteristic times can be introduced-the capture time into an unoccupied QD ensemble ${ }^{11}$ and the thermal escape time from an individual QD, 8,11

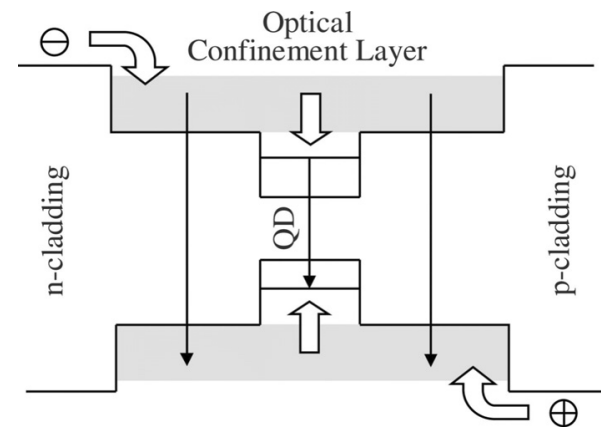

FIG. 1. Indirect injection into the active region of a QD laser. 


$$
\tau_{\text {capt }, 0}=\left(\sigma_{\mathrm{n}} \mathrm{v}_{\mathrm{n}} \frac{N_{\mathrm{S}}}{b}\right)^{-1}, \quad \tau_{\text {esc }}=\left(\sigma_{\mathrm{n}} \mathrm{v}_{\mathrm{n}} n_{1}\right)^{-1} .
$$

In a specific structure considered below, $\tau_{\text {capt }, 0}=1.63 \mathrm{ps}$ and $\tau_{\text {esc }}=0.07$ ps at $\sigma_{\mathrm{n}}=10^{-11} \mathrm{~cm}^{2}$.

We consider the spontaneous radiative recombination as the only mechanism of nonstimulated recombination in the OCL and QDs. The inclusion of the nonradiative Auger recombination will increase the threshold current density and the steady-state carrier density in the OCL, and, within the framework of the small-signal analysis, will decrease the differential nonstimulated recombination time, while not otherwise affecting the main derivations of this letter.

Our model does not include the wetting layer (WL), which is inherently present in self-assembled StranskiKrastanow grown QD structures. The WL can affect the carrier capture into QDs. In addition to the direct capture from the bulk OCL into QDs, carriers will also be captured from the OCL into the 2D WL and then from the WL into QDs. The inclusion of the WL will thus require a careful consideration of all these capture processes.

Due to inhomogeneous broadening of the transition energy in a QD-ensemble in an edge-emitting laser, we do not also consider the optical mode resonance with the QDtransition. This resonance in the context of QD nanocavity lasers was considered in Ref. 13.

Applying the small-signal analysis of rate equations, we consider the injection current density in Eq. (1) in the form of $j=j_{0}+\left(\delta j_{\mathrm{m}}\right) \exp (i \omega t)$, where $j_{0}$ is the dc component and the amplitude $\delta j_{\mathrm{m}}$ of the time-harmonic ac component is small $\left(\delta j_{\mathrm{m}} \ll j_{0}-j_{\text {th }}\right.$, where $j_{\text {th }}$ is the threshold current density). We correspondingly look for $n_{\mathrm{OCL}}, f_{\mathrm{n}}$, and $n_{\mathrm{ph}}$ in Eqs. (1)-(3) in the form of $n_{\mathrm{OCL}}=n_{\mathrm{OCL}, 0}+\left(\delta n_{\mathrm{OCL}-\mathrm{m}}\right) \exp (i \omega t), f_{\mathrm{n}}$ $=f_{\mathrm{n}, 0}+\left(\delta f_{\mathrm{n}-\mathrm{m}}\right) \exp (i \omega t)$, and $n_{\mathrm{ph}}=n_{\mathrm{ph}, 0}+\left(\delta n_{\mathrm{ph}-\mathrm{m}}\right) \exp (i \omega t)$, where $n_{\mathrm{OCL}, 0}, f_{\mathrm{n}, 0}$, and $n_{\mathrm{ph}, 0}$ are the solutions of the steadystate rate equations at $j=j_{0} .{ }_{10,11}$ In particular,

$$
f_{\mathrm{n}, 0}=\frac{1}{2}\left(1+\frac{\beta}{g^{\max }}\right)=\frac{1}{2}\left(1+\frac{1}{\tau_{\mathrm{ph}} \mathrm{V}_{\mathrm{g}} g^{\max }}\right),
$$

where the photon lifetime in the cavity is

$$
\tau_{\mathrm{ph}}=\frac{1}{\mathrm{v}_{\mathrm{g}} \beta}=\frac{L}{\mathrm{v}_{\mathrm{g}} \ln (1 / R)} .
$$

As seen from Eq. (5), the confined-carrier leveloccupancy $f_{\mathrm{n}, 0}$ in a QD at the steady-state is pinned at its threshold value and does not change with $j_{0}$ above the lasing threshold. In contrast to $f_{\mathrm{n}, 0}$, the steady-state free-carrier density $n_{\mathrm{OCL}, 0}$ in the OCL is not pinned-it rises with $j_{0}$ above the lasing threshold. It should be emphasized that it is the noninstantaneous capture of carriers from the OCL into QDs that causes this rise in $n_{\mathrm{OCL}, 0}$ above the lasing threshold. ${ }^{10,11}$

We obtain from Eqs. (1)-(3) a set of algebraic equations in the frequency-dependent small amplitudes $\delta n_{\mathrm{OCL}-\mathrm{m}}$, $\delta f_{\mathrm{n}-\mathrm{m}}$, and $\delta n_{\mathrm{ph}-\mathrm{m}}$, the solution of which yields the modulation response function $H(\omega)=\left|\delta n_{\mathrm{ph}-\mathrm{m}}(\omega) / \delta n_{\mathrm{ph}-\mathrm{m}}(0)\right|^{2}$. Finally, we arrive at a cubic equation for the square of the modulation bandwidth $\omega_{-3} \mathrm{~dB}$ - the frequency, at which $H(\omega)$ has fallen to half its dc $(\omega=0)$ value.

For an illustration of our results, room-temperature operation of a GaInAsP heterostructure lasing near $1.55 \mu \mathrm{m}$ (Ref. 8) is considered here. We assume 10\% QD-size fluctuations, the surface density of RDs in a single-layer $N_{\mathrm{S}}$

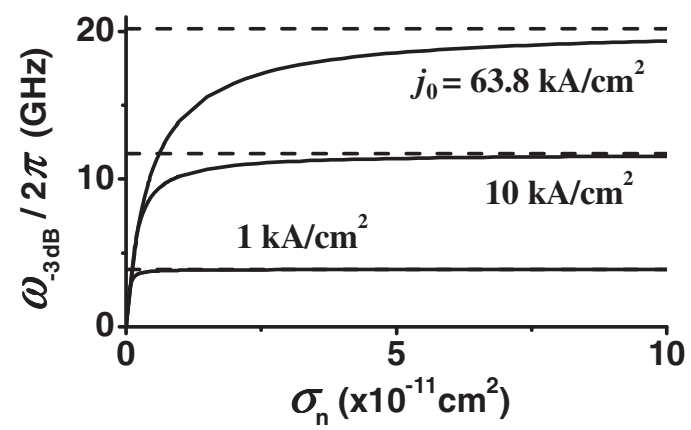

FIG. 2. Modulation bandwidth vs capture cross-section into a QD at different values of the dc component $j_{0}$ of the injection current density in a single-layer structure. The horizontal dashed lines show $\omega_{-3} \mathrm{~dB}$ for the case of instantaneous capture [Eq. 9 of Ref. 7]. $63.8 \mathrm{kA} / \mathrm{cm}^{2}$ is the optimum value of $j_{0}$ maximizing $\omega_{-3} \mathrm{~dB}$ for the case of instantaneous capture and, correspondingly, the top horizontal line shows $\omega_{-3 \mathrm{~dB}}^{\max }$ for that case [Eq. (11)]. $T=300 \mathrm{~K}$ and $L=1.1 \mathrm{~mm}$.

$=6.11 \times 10^{10} \mathrm{~cm}^{-2}$, and an ideal overlap between the electron and hole wave functions in a QD. At these parameters, the maximum modal gain in a single-QD-layer structure $g^{\max }=29.52 \mathrm{~cm}^{-1}$. The OCL thickness $b=0.28 \mu \mathrm{m}$ and the cavity length $L=1.1 \mathrm{~mm}$ (at this $L$ and the as-cleaved facet reflectivity $R=0.32$, the mirror loss $\beta=10 \mathrm{~cm}^{-1}$ ).

The modulation bandwidth depends strongly on the capture cross-section $\sigma_{\mathrm{n}}$. At a fixed $j_{0}$, with making slower the capture into QDs (reducing $\sigma_{\mathrm{n}}$ ), $\omega_{-3} \mathrm{~dB}$ decreases and finally becomes zero (Figs. 2 and 3 ).

As seen from Fig. 3, $\omega_{-3} \mathrm{~dB}=0$ at a certain nonvanishing value $\sigma_{\mathrm{n}}^{\mathrm{min}}$. This is due to the fact that, at a given $j_{0}$, no lasing is attainable in the structure if $\sigma_{\mathrm{n}}<\sigma_{\mathrm{n}}^{\mathrm{min}}$. Indeed, while $j_{0}$ is fixed, the threshold current density increases with decreasing $\sigma_{\text {n }}$ (the curve corresponding to the left axis),

$$
j_{\mathrm{th}}=\frac{e N_{\mathrm{S}}}{\tau_{\mathrm{QD}}} f_{\mathrm{n} 0}^{2}+e b B\left(n_{1} \frac{f_{\mathrm{n} 0}}{1-f_{\mathrm{n} 0}}+\frac{1}{\sigma_{\mathrm{n}} \mathrm{v}_{\mathrm{n}} \tau_{\mathrm{QD}}} \frac{f_{\mathrm{n} 0}^{2}}{1-f_{\mathrm{n} 0}}\right)^{2},
$$

where $f_{\mathrm{n}, 0}$ is given by Eq. (5). In order for the lasing to occur, $j_{0}$ should be higher than $j_{\text {th }}$. At a certain $\sigma_{\mathrm{n}}^{\min }, j_{\text {th }}$ becomes equal to $j_{0}$ (Fig. 3). At $\sigma_{\mathrm{n}} \leq \sigma_{\mathrm{n}}^{\min }, j_{\text {th }} \geq j_{0}$, which means that there can be no lasing and hence no direct modulation in the structure (the shaded region in Fig. 3).

The minimum tolerable $\sigma_{\mathrm{n}}$ for the lasing to occur at $j_{0}$ is found from the condition $j_{\text {th }}=j_{0}$ and is given by

$$
\sigma_{\mathrm{n}}^{\min }\left(j_{0}\right)=\frac{1}{\mathrm{v}_{\mathrm{n}} \tau_{\mathrm{QD}}} \frac{f_{\mathrm{n} 0}^{2}}{1-f_{\mathrm{n} 0}} \sqrt{e b B} \frac{\sqrt{j_{0}-\frac{e N_{\mathrm{S}}}{\tau_{\mathrm{QD}}} f_{\mathrm{n} 0}^{2}}+\sqrt{j_{\mathrm{th}}^{\mathrm{eq}}-\frac{e N_{\mathrm{S}}}{\tau_{\mathrm{QD}}} f_{\mathrm{n} 0}^{2}}}{j_{0}-j_{\mathrm{th}}^{\mathrm{eq}}},
$$

where $j_{\text {th }}^{\text {eq }}$ is $j_{\text {th }}$ for the case of instantaneous capture $\left[j_{\text {th }}^{\text {eq }}\right.$ is obtained using $\sigma_{\mathrm{n}}=\infty$ in Eq. (7)].

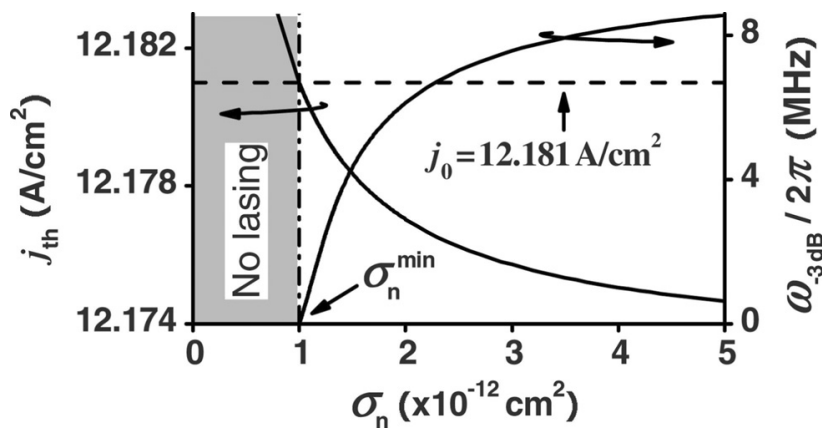

FIG. 3. Modulation bandwidth (at a very low $j_{0}$ ) and threshold current density vs capture cross-section into a QD in a single layer structure. 


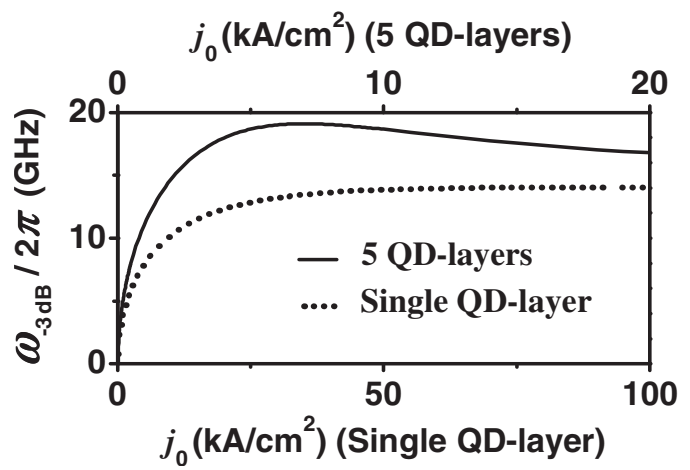

FIG. 4. Modulation bandwidth vs dc component of the injection current density in single- and 5-QD-layer-structures. A plausible value $\sigma_{\mathrm{n}}$ $=10^{-11} \mathrm{~cm}^{2}$ (Refs. 14 and 15) of the capture cross-section is used.

As seen from Eq. (8), when $j_{0}$ decreases and approaches $j_{\mathrm{th}}^{\mathrm{eq}}, \sigma_{\mathrm{n}}^{\mathrm{min}}$ increases infinitely, i.e., no lasing is attainable at $j_{0} \leq j_{\text {th }}^{\text {eq }}$ even if the carrier capture into QDs is instantaneous.

With increasing $j_{0}, \sigma_{\mathrm{n}}^{\min }$ becomes smaller, i.e., the lasing can occur and hence the direct modulation of the output power is possible at a slower capture. At high $j_{0}$ (when $\left.\sigma_{\mathrm{n}}^{\min } \rightarrow 0\right)$, the asymptotic expression for $\omega_{-3 \mathrm{~dB}}$ for $\sigma_{\mathrm{n}}$ in the vicinity of $\sigma_{\mathrm{n}}^{\min }\left[\left(\sigma_{\mathrm{n}}-\sigma_{\mathrm{n}}^{\min }\right) / \sigma_{\mathrm{n}}^{\min } \ll 1\right]$ is

$$
\omega_{-3 \mathrm{~dB}} \approx 2 \sqrt{r-1} \mathrm{v}_{\mathrm{g} g} g \frac{f_{\mathrm{max}}\left(1-f_{\mathrm{n} 0}\right)}{2-f_{\mathrm{n} 0}} \frac{\sigma_{\mathrm{n}}-\sigma_{\mathrm{n}}^{\min }}{\sigma_{\mathrm{n}}^{\min }},
$$

where the numerical parameter $r=10^{0.3} \approx 1.995$ originates from the definition of the $-3 \mathrm{~dB}$ bandwidth, $10 \log _{10} H\left(\omega_{-3} \mathrm{~dB}\right)=-3$.

As a function of the dc component $j_{0}$ of the injection current density, $\omega_{-3} \mathrm{~dB}$ has a maximum (Fig. 4). In a singleQD-layer structure (the dotted curve), the optimum value $j_{\text {opt }}$ of $j_{0}$, at which $\omega_{-3}^{\max }$ iB is attained, is very high, i.e., $\omega_{-3 \mathrm{~dB}}^{\max }$ is unattainable. As seen from the figure, there are the following two advantages in a multi-QD-layer structure (the solid curve) as compared to a single-layer structure: (i) $\omega_{-3 \mathrm{~dB}}$ is considerably higher at the same $j_{0}$ and (ii) $j_{\text {opt }}$ is considerably reduced, which means that $\omega_{-3 \mathrm{~dB}}^{\max }$ is practically attainable.

At large $\sigma_{\mathrm{n}}$, when $\tau_{\text {capt }, 0} / \tau_{\text {ph }} \ll 1\left[\tau_{\text {capt }, 0}\right.$ and $\tau_{\text {ph }}$ are given by Eqs. (4) and (6), respectively], both $\omega_{-3 \mathrm{~dB}}$ at a given $j_{0}$ (Fig. 2) and $\omega_{-3 \mathrm{~dB}}^{\max }$ (Fig. 5) asymptotically approach their saturation values (the horizontal dashed lines) corresponding to the case of instantaneous capture into QDs,

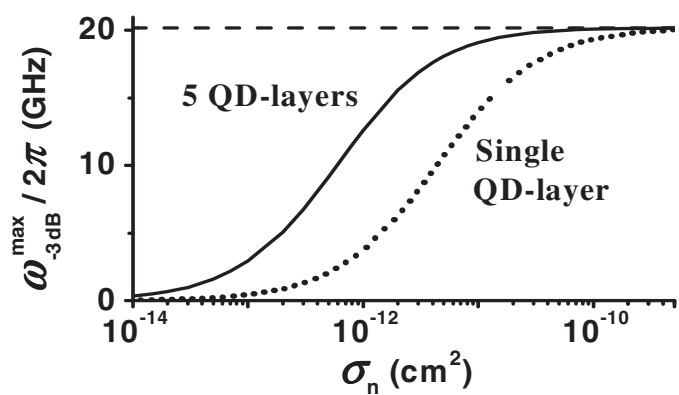

FIG. 5. Maximum modulation bandwidth vs capture cross-section into a QD. The horizontal dashed line shows $\omega_{-3 \mathrm{~dB}}^{\max }$ for the case of instantaneous capture into QDs [Eq. (11)].

$$
\begin{gathered}
\left(\left.\omega_{-3 \mathrm{~dB}}\right|_{\sigma_{\mathrm{n}}=\infty}-\omega_{-3 \mathrm{~dB}}\right), \quad\left(\left.\omega_{-3 \mathrm{~dB}}^{\max }\right|_{\sigma_{\mathrm{n}}=\infty}-\omega_{-3 \mathrm{~dB}}^{\max }\right) \\
\propto \frac{\tau_{\mathrm{capt}, 0}}{\tau_{\mathrm{ph}}} \propto \frac{1}{\sigma_{\mathrm{n}}},
\end{gathered}
$$

where $\left.\omega_{-3 \mathrm{~dB}}\right|_{\sigma_{\mathrm{n}}=\infty}$ is given by Eq. 9 of Ref. 7 and

$$
\left.\omega_{-3 \mathrm{~dB}}^{\max }\right|_{\sigma_{\mathrm{n}}=\infty} \approx \frac{\sqrt{2}}{\tau_{\mathrm{ph}}} \text {. }
$$

As seen from Fig. 5, while the saturation value of $\omega_{-3}^{\max } \mathrm{dB}$ at $\sigma_{\mathrm{n}} \rightarrow \infty$ and at a fixed $L$ [Eq. (11)] does not depend on the number of QD-layers, $\omega_{-3 \mathrm{~dB}}^{\max }$ at a given finite $\sigma_{\mathrm{n}}$ is higher in a multilayer structure as compared to a single-layer structure.

In conclusion, we have shown that the carrier capture from the OCL into QDs can strongly limit the modulation bandwidth $\omega_{-3} \mathrm{~dB}$ of a QD laser. $\omega_{-3} \mathrm{~dB}$ is highest in the case of instantaneous capture into QDs, when the cross-section of carrier capture into a QD $\sigma_{\mathrm{n}}=\infty$. With reducing $\sigma_{\mathrm{n}}, \omega_{-3 \mathrm{~dB}}$ decreases and becomes zero at a certain nonvanishing $\sigma_{\mathrm{n}}^{\min }$. This $\sigma_{\mathrm{n}}^{\min }$ presents the minimum tolerable $\sigma_{\mathrm{n}}$ for the lasing to occur at a given dc component $j_{0}$ of the injection current density. The use of multiple-layers with QDs has been shown to significantly improve the modulation response of the laser- $\omega_{-3 \mathrm{~dB}}$ is considerably higher in a multilayer structure as compared to a single-layer structure at the same $j_{0}$. At a plausible cross-section $\sigma_{\mathrm{n}}=10^{-11} \mathrm{~cm}^{2},{ }^{14,15} \omega_{-3 \mathrm{~dB}}$ as high as $19 \mathrm{GHz}$ can be obtained in a 5-QD-layer structure with the cavity length $L=1.1 \mathrm{~mm}$ at a practical value of $j_{0}$ $=7 \mathrm{kA} / \mathrm{cm}^{2}$. Our analysis provides a basis for optimizing the QD laser design for high-speed operation.

L.V.A. and Y.W. acknowledge the U.S. Army Research Office (Grant No. W911-NF-08-1-0462), Y.W. also acknowledges the China Scholarship Council, and R.A.S. acknowledges the Russian Foundation for Basic Research (Grant No. 08-02-01337) and the Program "Fundamental Research in Nanotechnology and Nanomaterials" of the Presidium of the Russian Academy of Sciences for support of this work.

${ }^{1}$ R. Dingle and C. H. Henry, U.S. Patent No. 3982207 (21 September 1976).

${ }^{2}$ Zh. I. Alferov, Rev. Mod. Phys. 73, 767 (2001).

${ }^{3}$ W. T. Tsang, Appl. Phys. Lett. 40, 217 (1982).

${ }^{4}$ P. S. Zory, Jr., Quantum Well Lasers (Academic, Boston, 1993).

${ }^{5}$ Y. Arakawa and H. Sakaki, Appl. Phys. Lett. 40, 939 (1982).

${ }^{6}$ A. E. Zhukov, A. R. Kovsh, S. S. Mikhrin, A. P. Vasil'ev, E. S. Semenova, N. A. Maleev, V. M. Ustinov, M. M. Kulagina, E. V. Nikitina, I. P. Soshnikov, Y. M. Shernyakov, D. A. Livshits, N. V. Kryjanovskaya, D. S. Sizov, M. V. Maximov, A. F. Tsatsul'nikov, N. N. Ledentsov, D. Bimberg, and Zh. I. Alferov, Physica E 17, 589 (2003).

${ }^{7}$ L. V. Asryan and R. A. Suris, Appl. Phys. Lett. 96, 221112 (2010).

${ }^{8}$ L. V. Asryan and R. A. Suris, Semicond. Sci. Technol. 11, 554 (1996).

${ }^{9}$ L. V. Asryan and R. A. Suris, IEEE J. Quantum Electron. 34, 841 (1998).

${ }^{10}$ L. V. Asryan, S. Luryi, and R. A. Suris, Appl. Phys. Lett. 81, 2154 (2002).

${ }^{11}$ L. V. Asryan, S. Luryi, and R. A. Suris, IEEE J. Quantum Electron. 39, 404 (2003).

${ }^{12}$ R. Nagarajan, M. Ishikawa, T. Fukushima, R. S. Geels, and J. E. Bowers, IEEE J. Quantum Electron. 28, 1990 (1992).

${ }^{13}$ M. Lorke, T. R. Nielsen, and J. Mørk, Appl. Phys. Lett. 97, 211106 (2010)

${ }^{14}$ O. Engström, M. Kaniewska, Y. Fu, J. Piscator, and M. Malmkvist, Appl. Phys. Lett. 85, 2908 (2004).

${ }^{15}$ S. K. Zhang, H. J. Zhu, F. Lu, Z. M. Jiang, and X. Wang, Phys. Rev. Lett. 82, 2622 (1999). 